\title{
VISIBILIZANDO LA VIOLENCIA HACIA LAS MUJERES EN EL EMBARAZO
}

\section{Josefina Goberna-Tricas}

Departamento de Enfermería de Salud Pública, Salud Mental y Maternoinfantil. ADHUC, centro de investigación en Teoría, Género y Sexualidad.

Universitat de Barcelona

Correo electrónico: jgoberna@ub.edu

Relacionar las palabras embarazo y violencia puede parecer contradictorio puesto que en el imaginario colectivo se suele asociar el nacimiento de los hijos y el embarazo con plenitud y realización personal. Sin embargo, algunos estudios nos indican que hasta un $20 \%$ de las mujeres pueden sufrir violencia, por parte de la pareja, durante el embarazo, sea violencia física, psicológica, económica o sexual. Por ello, debemos plantearnos si los profesionales sanitarios en general y las matronas en particular, como responsables del seguimiento del embarazo normal, tienen las competencias necesarias para la detección y abordaje de estas situaciones. Podemos preguntarnos: ¿Desde los ámbitos educativos, desde la Universidad, impartimos una formación adecuada en este sentido? Sabemos que facultades universitarias y centros sanitarios han implantado programas formativos al respecto, dando cumplimiento a la Ley Medidas de Protección Integral contra la violencia de género de 2004, pero ello no significa que las y los profesionales se sientan empoderadas/os en este campo de actuación. No es fácil preguntar o acompañar a aquellas mujeres en las que se detecta alguna de estas situaciones, especialmente teniendo en cuenta que la formación en los ámbitos sanitarios sigue teniendo un fuerte sesgo biologista. En este número de Musas: Revista de Investigación en Mujer, Salud y Sociedad, se presenta una aproximación a las repercusiones y efectos de la violencia de género sobre el feto, presentado en el artículo de Gregorio junto con 
dos propuestas formativas dirigidas a profesionales sanitarios elaboradas con seriedad y rigor y presentadas por Paya et al. y por Carmona del Rio.

Pero si el abordaje de la violencia de género durante el embarazo es un tema sensible, ello aún se manifiesta con mayor fuerza cuando es el propio sistema sanitario el generador de violencia hacia las mujeres, en lo que se conoce como «violencia obstétrica», un término que denuncia el exceso de medicalización en la atención sanitaria al proceso fisiológico del parto y el trato deshumanizado dado a las mujeres durante dicha atención. La expresión «violencia obstétrica» sorprende a muchas personas e incómoda a algunos profesionales sanitarios. A pesar de ello, su uso se ha extendido en los últimos años. Podemos preguntamos: ¿Es adecuado el término violencia obstétrica para definir esta situación? ¿Podríamos usar otros términos como maltrato, poder obstétrico o violencia institucional? ¿La medicalización de un proceso natural como el parto puede ser considerada, per se, como violencia obstétrica? ¿Solamente si esta medicalización se ha llevado a cabo sin la adecuada información y consentimiento por parte de la mujer? ¿Debemos considerar la violencia obstétrica como una forma de violencia de género? Algunas de estas cuestiones se responden en este número, en que se presenta, por parte de Boladeras, un análisis de los conceptos de violencia estructural o institucional y violencia cultural o simbólica, así como la revisión de algunas de sus manifestaciones sociológicas, jurídicas y éticas. Un análisis estadístico de casos en que las mujeres afirman haber padecido violencia obstétrica es presentado por Iglesias et al., para finalmente encontrarnos con un estudio de asociación entre variables sociodemográficas y parto por cesárea (algunas de ellas posiblemente catalogables como violencia obstétrica) presentado por Benlloch et al.

Los trabajos presentados en este número derivan de las aportaciones al «III Congreso Internacional Género, Ética y Cuidado: Visibilizando la violencia hacia las mujeres en el embarazo», realizado en Barcelona en mayo de 2018 y organizado en el marco del Proyecto de investigación 2016ACUP083 «Prevención y detección de les violencias de género durante el embarazo: de la violencia machista a la violencia obstétrica». Los 144 asistentes al congreso debatimos sobre todos estos aspectos y, a lo largo de este año 2019, tanto en este número como en el siguiente, la revista MUSAS publicará algunas de las ponencias y comunicaciones más destacadas del congreso. 